\title{
Les apports et les limites liés aux pratiques inclusives et la place de la collaboration dans ces pratiques: une métasynthèse
}

\section{Nadia Rousseau, Mathieu Point, Raymond Vienneau, Marie-Élaine Desmarais et Karelle Desmarais}

Cet article découle des résultats d'une métasynthèse qui avait comme principal objectif de produire une nouvelle compréhension des enjeux de l'intégration et de l'inclusion scolaires des élèves ayant des besoins particuliers du primaire et du secondaire en classe ordinaire. L'article présente, dans un premier temps, les résultats de la métasynthèse eu égard aux apports et aux limites associés aux pratiques qui tendent vers l'inclusion scolaire. Dans un deuxième temps, il s'intéresse aux pratiques collaboratives, une condition essentielle au développement de pratiques plus inclusives. Trois dimensions de la collaboration sont explorées, soit la collaboration avec et par les pairs, la collaboration avec et entre les professionnels, et la collaboration avec les parents.

\section{Introduction}

Comme en témoignent Rousseau, Bergeron et Vienneau (2013), «'inclusion scolaire continue d'alimenter de nombreuses questions de recherche et de développement en éducation, dont les problématiques associées à la conceptualisation même du phénomène, à ses postulats, à ses aspects opérationnels et à ses conditions de mise en œuvre» (p.72). Outre ces défis conceptuels et théoriques, d'autres aspects opérationnels retiennent l'attention, et ce, sous différents angles (p. ex., l'organisation des modèles de services, l'attitude des enseignants, le leadership de la direction d'école et l'accompagnement des intervenants scolaires). C'est dans cette lignée que s'inscrit notre réflexion sur la prise en compte de la diversité en classe ordinaire au primaire et au secondaire. Cet article fait état des constats qui émergent d'une métasynthèse, notamment eu égard aux apports et limites des pratiques qui tendent vers l'inclusion scolaire et à la place qu'occupe la collaboration au sein de ces pratiques. 


\section{Intégration et inclusion scolaires: enjeux de ces réalités multiples}

Bien que de nombreuses études aient mis en lumière des liens opérationnels entre les écoles pratiquant une pédagogie de l'inclusion et les écoles dites efficaces (Gallucci, 1997; Morefield, 2002; Slee \& Weiner, 2001) de même qu'entre les pratiques inclusives et l'apprentissage et le développement social (Vienneau, 2004, 2010), il serait faux de prétendre qu'une majorité d'écoles adoptent de telles pratiques. Comme en témoignent Gardou, Poizat et Audureau (2004), les projets d'inclusion scolaire restent des exceptions, alors qu'une majorité d'écoles continuent d'adopter des pratiques qui reposent davantage sur des exigences normatives associées à la perspective de l'intégration scolaire. Cette perspective renvoie au désir de combattre la marginalisation ou l'exclusion des élèves dits handicapés (Bergeron \& St-Vincent, 2011) en plaçant ces derniers dans un cheminement scolaire le plus près possible du cheminement prévu pour les élèves sans handicap (AuCoin \& Vienneau, 2014). Pour certains, dont Bourdieu (1993), cette perspective peut faire de ces élèves dits handicapés des «exclus de l'intérieur». La perspective d'inclusion s'appuie davantage sur la notion de situation de handicap, où la place accordée aux caractéristiques individuelles est réduite pour offrir une plus grande importance à d'autres facteurs d'influence comme l'environnement dans lequel la personne évolue (Fougeyrollas, 2010). Cette perspective renvoie également à la notion de diversité apprenante, souvent liée à une vision plus positive de la différence, soit comme une richesse et une possibilité de réduire les inégalités et de maintenir la justice sociale (AuCoin \& Vienneau, 2014; Crahay, 2000; Lebeaume \& Coquidé, 2002; Morvan, 2015; Talbot, 2011). L'ouvrage de Bélanger et Duchesne (2010) contribue à la mise en évidence de réalités multiples en matière d'interventions inclusives, tant en contexte canadien qu'en contexte européen. Ainsi, l'identification des enjeux de l'inclusion scolaire gagne à faire l'objet d'une recension d'écrits systématique à travers la méthode de métasynthèse ${ }^{1}$. Deux objectifs spécifiques retiennent l'attention: 1) Identifier les apports et les limites de l'inclusion scolaire, eu égard à l'apprentissage et au développement social des élèves à risque ${ }^{2}$ et des élèves ayant des besoins particuliers ${ }^{3}$ du primaire et du secondaire ${ }^{4}$. 2) Identifier les conditions favorables et défavorables à l'inclusion scolaire des élèves à risque et des élèves ayant des besoins particuliers, eu égard à l'organisation des services à l'élève et aux pratiques pédagogiques. Ce sont les résultats associés à ces deux objectifs spécifiques qui sont ici présentés.

\section{Métasynthèse}

Pour atteindre les objectifs de cette synthèse de connaissances, l'analyse de données secondaires est utilisée, soit la métasynthèse (Beaucher \& Jutras, 2007; Erwin, 
Brotherson \& Summers, 2011; Walsh \& Downe, 2005), qui recourt à l'analyse des résultats de recherches qualitatives. Cette méthode d'analyse contribue à une compréhension plus poussée d'un phénomène particulier menant à l'émergence de «conclusions nouvelles» (Beaucher \& Jutras, 2007, p. 63). Fidèle aux étapes de la métasynthèse (Littell, Campbell, Green \& Toews, 2008; Petticrew \& Roberts, 2006; Walsh \& Downe, 2005), les textes recensés (2000 à 2015) et analysés résultent d'un travail rigoureux: identification des critères d'éligibilité; identification des descripteurs - anglais et français - spécifiques à l'objet d'étude; consultation de nombreuses bases de données; évaluation de la qualité des études recensées à l'aide d'une liste de critères de qualité définis pour la métasynthèse (Campbell et al., 2003) et extraction des données pour analyse thématique. Pour être retenues, les études devaient porter sur l'intégration ou l'inclusion scolaires; l'apprentissage, le développement social, l'organisation des services, les pratiques pédagogiques, les conditions favorables et défavorables à l'intégration et l'inclusion; le primaire ou le secondaire; et les élèves dits à risque (Ministère de l'Éducation, du Loisir et du Sport [MELS], 2007). Ces études devaient également être des études primaires de langue exclusivement française ou anglaise et uniquement publiées à partir de 2003. Les critères d'évaluation de la qualité des études recensées comprenaient: méthode (protocole, échantillon, déroulement de l'étude et analyse de données); résultats (crédibilité, contribution à l'avancement des connaissances, respect des objectifs ou buts de la recherche et possibilité de généralisation); discussion (clarté et cohérence de la présentation des constats, réflexivité, neutralité, vérifiabilité et considérations éthiques).

\section{Principaux résultats}

La métasynthèse aura permis le recensement initial de 270 écrits. De ce nombre, seuls 51 écrits $(18,8 \%)$ répondaient aux critères d'éligibilité et de qualité, soit 42 articles scientifiques, 7 thèses et 2 chapitres de livre. La proportion de textes retenus est similaire à celle d'autres études adoptant la métasynthèse ou la méta-analyse (Vienneau, 2010). L'analyse de ces écrits permet de porter un nouveau regard sur les principaux apports et limites associés aux pratiques qui tendent vers l'inclusion scolaire. Elle met aussi en évidence la place centrale qu'occupe la collaboration dans le développement de pratiques plus inclusives.

\section{Apports et limites des pratiques qui tendent vers l'inclusion scolaire}

En réponse au premier objectif de recherche et à la suite de l'analyse thématique des écrits retenus, des apports et des limites sont identifiés en lien aux pratiques qui tendent vers l'inclusion scolaire (perspective centrée sur la diversité). Les lignes qui suivent explicitent les apports recensés en lien aux thèmes de l'apprentissage, de la socialisation, de l'engagement et du sentiment d'appartenance, de même que les limites répertoriées. 


\section{Apprentissage}

La métasynthèse révèle que les pratiques qui tendent vers l'inclusion scolaire sont associées à des retombées positives sur le plan de l'apprentissage. L'étude de Nootens (2010) précise que les élèves ayant des besoins particuliers (difficultés de langage ${ }^{5}$ ) en contexte d'inclusion améliorent leur rendement en lecture et en écriture, de même que leur autonomie. Cela s'observe par le développement de leur sensibilité à vérifier l'orthographe des mots de leurs productions écrites, à structurer les idées qu'ils veulent exprimer et à conserver leur environnement de travail propre et ordonné. Ces progrès apportent des retombées positives sur les apprentissages des élèves, étant plus aptes à bien travailler seuls, à démontrer une attitude plus positive à l'égard des tâches à réaliser et à s'engager plus activement en classe, et ce, malgré la grande diversité des classes. Idol (2006) précise que les pratiques plus inclusives au primaire influencent l'obtention de meilleurs résultats aux tests nationaux. Ce même constat ne s'applique toutefois pas au niveau secondaire, où les résultats demeurent relativement semblables.

Kartsen, Peetsma, Roeleveld et Verger (2001) comparent le développement psychosocial et académique des élèves à risque du primaire se trouvant en classe ordinaire à ceux de la classe spéciale. Ils arrivent à la conclusion que ceux de la classe ordinaire sont avantagés comparativement à ceux de la classe spéciale, avec un meilleur rendement dans les habiletés langagières et mathématiques. Plus les élèves gagnent en âge, plus l'écart entre les deux groupes se fait sentir. L'étude met aussi en évidence que le bien-être et le développement psychosocial des élèves, notamment l'estime de soi, les comportements sociaux, l'attitude au travail, la santé et le support reçu à la maison, seraient selon les enseignants et les parents, plus favorables pour ceux de la classe ordinaire que pour ceux de la classe spéciale. Cela dit, les enseignants de la classe spéciale, qui s'associent à d'autres experts du milieu scolaire et qui se dotent d'un plan d'action, favorisent le développement des habiletés langagières de même que l'attitude au travail des élèves à risque de ce type de classe.

Quant aux limites recensées dans la métasynthèse, les pratiques plus inclusives nécessitent de la vigilance de la part des différents acteurs scolaires, puisque l'absence de toute forme de différenciation risque fort de mener les élèves les plus vulnérables à l'échec. Mowat (2009) précise que chez les élèves à risque (difficultés sociale, émotionnelle et comportementale) du secondaire, la fréquentation d'une classe ordinaire n'entraîne pas nécessairement une augmentation du taux de réussite aux tests nationaux. La réussite de ces tests constitue un bel exemple de comparaison à la norme, ce que déplore AuCoin (2010).

\section{Socialisation}

O'Rourke et Houghton (2009) constatent que la présence des pairs en contexte inclusif et au niveau secondaire a une influence positive sur le développement social des élèves ayant des besoins particuliers (déficience intellectuelle modérée), ce qui contribuerait à l'augmentation de leur motivation scolaire. D'ailleurs, 
Mowat (2009) remarque une diminution des comportements d'opposition de même qu'une amélioration des comportements chez certains élèves ayant des besoins particuliers (déficience intellectuelle modérée) dans les écoles qui tendent vers des pratiques plus inclusives. Pour leur part, Groom et Rose (2005) ont pu noter des résultats probants lorsque des aides-enseignants ${ }^{6}$ étaient déployés en classe inclusive. Entre autres, les élèves à risque (difficultés sociales, émotionnelles et comportementales) âgés de 7 à 11 ans se retrouvaient plus rarement en retrait, leur besoin d'avoir un soutien individuel était moindre et ils avaient davantage le sentiment de faire partie de la communauté. Quant à Nowicki et Brown (2013), ils font ressortir que lorsque les élèves ordinaires du primaire âgés de 9 à 12 ans sont informés des défis scolaires que rencontrent leurs pairs et des raisons justifiant leurs sorties occasionnelles de la classe pour recevoir une aide particulière, ils s'impliquent davantage dans le processus d'inclusion de ces élèves.

En ce qui a trait aux limites recensées dans la métasynthèse, Mowat (2009) stipule que les enseignants en contexte plus inclusifs sont ambivalents quant au succès de leurs interventions. Pour quelques-uns de leurs élèves du secondaire, il est évident que ces interventions ont eu un impact positif sur leurs comportements, tandis que pour d'autres, l'amélioration est moindre. L'auteure estime qu'il existe une étroite relation entre le comportement des élèves et les particularités des contextes. Elle observe également une relation entre les difficultés d'apprentissage et le comportement puisque ce sont les élèves qui parviennent difficilement à réaliser une tâche qui ont tendance à adopter une attitude dérangeante au sein de la classe. Par ailleurs, l'étude de Kelly et Norwich (2004) permet de souligner le fait que les élèves à risque (trouble d'apprentissage) sont fréquemment «étiquetés» en raison de leur différence.

\section{Engagement et sentiment d'appartenance}

La métasynthèse met en évidence que les pratiques plus inclusives semblent favorables au développement d'une meilleure compréhension par les enseignants et par les pairs des raisons pour lesquelles les élèves manifestent des comportements dérangeants en classe (Mowat, 2009). En effet, les pairs se montrent attentifs aux différentes difficultés que vivent les élèves à risque (trouble d'apprentissage) et ils sont sensibles au fait qu'il est inapproprié d'avoir des comportements agressifs à leur égard (Nowicki \& Brown, 2013). Cette conscientisation a pour effet de réduire le phénomène d'intimidation qui a lieu au primaire entre les élèves ordinaires et les élèves à risque (trouble d'apprentissage). AuCoin (2010) ajoute que cela favorise également le développement d'une attitude positive face à la différence entre les pairs puisque même les élèves ordinaires peuvent réaliser qu'ils ont eux-mêmes leurs propres particularités.

Il est intéressant de constater à la lumière des écrits retenus qu'en contexte plus inclusif, tous les acteurs du milieu scolaire s'unissent et travaillent ensemble dans le but d'améliorer le développement de l'élève, ce qui entraîne des 
retombées positives sur chacun des professionnels du milieu, dont le personnel scolaire qui adopte une attitude plus positive au travail (Boyle, Topping, JundalSnape \& Norwich, 2012). Idol (2006), pour sa part, s'est intéressée à l'impact des pratiques plus inclusives sur les autres élèves de la classe. Il ressort de son étude que, pour la majorité des élèves $(68 \%)$, un progrès est perçu au niveau du rendement scolaire (36\%), ou la performance demeure stable (32\%). Des constats similaires sont relevés en ce qui a trait aux comportements sociaux envers les élèves ayant des difficultés. Ce dernier résultat converge avec celui de l'étude de Nootens (2010), qui rend compte d'une amélioration des comportements des élèves et de l'organisation de la classe (autonomie, attitude plus positive quant aux tâches à réaliser, participation plus grande en classe et dans l'accomplissement des travaux scolaires).

Bien qu'aucune limite spécifique à l'engagement des élèves ne soit répertoriée dans les écrits retenus, d'autres défis retiennent l'attention. En contexte d'inclusion scolaire au secondaire, Picon (2010) met en doute l'accès sans restriction aux dossiers personnels des élèves ayant des besoins particuliers (déficience intellectuelle modérée à sévère). Selon l'auteure, cette pratique remet en question le principe de confidentialité auquel ces enfants ont droit. Afin de respecter cet espace privé, les acteurs de l'éducation pourraient avoir uniquement accès aux parties du dossier les concernant. L'auteure remarque également un certain manque de délicatesse dans le discours des acteurs de l'éducation à l'égard de leurs élèves. Ainsi, ils tendent à utiliser des qualificatifs qui figent l'image personnelle des jeunes, et ce, même en leur présence. Par exemple, une enseignante pourrait présenter une élève de sa classe à l'orthopédagogue comme étant l'«hyperactive» et un autre comme étant le «lunatique».

\section{Collaboration: condition essentielle au développement de pratiques plus inclusives}

En réponse au deuxième objectif de recherche et à la suite de l'analyse thématique des écrits retenus, il a été possible de relever que parmi les conditions favorables au développement de pratiques plus inclusives, la collaboration et la culture de collaboration occupent une place prédominante.

\section{Pratiques collaboratives}

La métasynthèse montre que les pratiques collaboratives constituent une condition sine qua non de l'inclusion scolaire. Dans ce contexte, l'inclusion se traduit par l'engagement de tous les acteurs de l'éducation vers une même mission, soit l'apprentissage, plutôt que le rassemblement de tous ces acteurs sous un même toit (Rogers, 2007). Trois dimensions de collaboration ressortent, soit: 1) avec et par les pairs, 2) avec et entre les professionnels, et 3) avec les parents. 
Avec et par les pairs: La métasynthèse révèle que les pratiques plus inclusives misent sur la participation de l'ensemble des élèves de la classe lors des activités d'apprentissage. L'étude de Combs, Elliott et Whipple (2010) met en évidence que les enseignants qui adoptent des pratiques plus inclusives n'hésitent pas à exploiter le pairage d'un élève à risque ou ayant des besoins particuliers à un ou des pairs ordinaires, ce qui se révèle aussi bénéfique pour celui qui aide que pour celui qui apprend. O'Rourke et Houghton (2009) en arrivent d'ailleurs aux mêmes constats. Pour leur part, Lee et Low (2013) mettent en évidence le soutien par les pairs en classe inclusive, notamment par l'exploitation de sous-groupes d'élèves, où chaque sous-groupe est composé d'une diversité d'apprenants. Ce mode de fonctionnement rend possible l'enseignement réciproque entre pairs, est associé à des retombées positives pour tous les élèves de la classe, en plus de contribuer au développement des habiletés de coopération chez ces derniers. En complément, Lee et Low (2013) constatent, que plus les élèves ordinaires sont exposés à un jeune âge aux élèves à risque (troubles d'apprentissage), plus il est naturel pour eux d'être généreux et tolérants à leur égard. Idol (2006) suggère fortement d'informer l'ensemble des élèves de la classe des raisons pour lesquelles certains de leurs pairs ayant des besoins particuliers nécessitent une aide particulière ou pourquoi certaines tâches sont adaptées.

Dans le cas de la réintégration d'élèves à risque (difficultés comportementales, émotionnelles et sociales), Pilay, Dunbar-Krige et Mostert (2013) et Dubé (2008) suggèrent de mettre en place des groupes de pairs pouvant jouer le rôle de mentors auprès de ces élèves. Les auteurs démontrent que le pairage d'un élève réintégré à un élève mentor contribue à rendre l'expérience de la réintégration plus positive. Outre le travail scolaire appuyé par les pairs, il est fort intéressant de constater que la culture d'entraide et de collaboration entre pairs donne lieu à des actions qui débordent de la salle de classe. Ainsi, interrogés sur les stratégies contribuant à la participation des élèves à risque ou ayant des besoins particuliers à l'ensemble des activités de l'école, les élèves ordinaires affirment aider, encourager et soutenir les élèves à risque ou ayant des besoins particuliers, leur donner des trucs et des explications, agir comme modèles dans la classe, travailler en sous-groupes avec tous les élèves de la classe, les intégrer aux clubs de l'école, voire créer de nouvelles activités parascolaires pour rejoindre les forces et les intérêts de ces élèves (Nowicki \& Brown, 2013). La même étude révèle que les élèves ordinaires estiment que l'attitude de leur enseignant peut nuire ou contribuer à la réelle participation des élèves à risque ou ayant des besoins particuliers en classe et à la récréation. Ainsi, un enseignant qui met en valeur les similitudes entre élèves plutôt que leurs différences agirait positivement sur la culture d'entraide et de collaboration dans la classe. De plus, certains enseignants contribueraient à faire connaître et comprendre aux élèves ordinaires les défis que vivent certains de leurs pairs. Cette connaissance et cette compréhension contribueraient à une meilleure participation sociale entre pairs. Enfin, cette même étude met de l'avant que les enseignants qui expriment clairement 
que l'intimidation entre pairs n'est pas acceptable contribueraient à la prise de position des pairs ordinaires pour défendre, soutenir ou dénoncer l'intimidation à l'égard de leurs pairs.

Avec et entre les professionnels: Les travaux consultés aux fins de la métasynthèse mettent clairement en évidence que les enseignants adoptant une pratique plus inclusive n'hésitent pas à collaborer avec différents professionnels. Selon Groom et Rose (2005), l'aide-enseignant au primaire agit auprès de l'apprenant lui-même, de l'enseignant, et plus largement au sein de l'école. Les auteurs identifient des caractéristiques clés chez ce professionnel permettant d'optimiser la qualité de l'aide apportée: patience, habiletés de résolution de problèmes, bonne écoute, excellente capacité d'adaptation, aptitude au travail en équipe, désir d'apprendre, capacité de travailler sous pression, sens de la justice et compréhension accrue des besoins des élèves. L'étude met également en évidence que l'aide-enseignant disposera de temps pour établir des liens significatifs avec les élèves. Ainsi, il pourra s'investir auprès des élèves dans deux fonctions complémentaires. La première, comme agent de soutien individuel à travers des activités de petits groupes, de l'aide à l'apprentissage, de la mise en place d'un système d'émulation, et de la participation à des moments consacrés au dialogue. La deuxième fonction complémentaire, comme agent de liaison entre l'école et la famille à travers le mentorat, le soutien à l'heure du dîner, le soutien aux récréations et la participation à des groupes de soutien entre pairs (développement émotionnel, estime de soi, habiletés sociales, etc.). Ces auteurs reconnaissent également à l'aide-enseignant un apport considérable dans la planification d'activités de petits groupes, soit l'établissement de liens avec les parents et d'autres professionnels externes à l'école ainsi que l'aide à l'élève dans la résolution de conflits. Précisons que l'ensemble des actions posées par ce professionnel gagnent à s'inscrire dans une démarche collaborative avec l'enseignant (co-planification, discussion sur le progrès des élèves, etc.) (Groom \& Rose, 2005; O’Rourke \& Houghton, 2009; Toullec-Théry \& Nédélec-Trohel, 2010). L'étude de Logan (2006), centrée sur la perception des élèves étant accompagnés par un aide-enseignant, confirme également l'apport indéniable de ce professionnel en contexte inclusif, notamment en ce qui a trait à la réalisation des travaux scolaires, au développement de comportements plus adéquats et au développement de relations d'amitié. Quant aux parents de ces élèves, l'étude révèle également qu'ils sont favorables à la présence de l'aide-enseignant, et ce, tant en classe que dans la cour de récréation. Ils estiment que ce professionnel contribue favorablement à l'apprentissage, aux comportements et aux interactions sociales de leurs enfants.

Morrison et Burgman (2009) se sont pour leur part intéressées au rôle de l'ergothérapeute en soutien à l'inclusion scolaire, notamment pour les élèves ayant des besoins particuliers (déficiences intellectuelle, motrice, auditive et visuelle). Elles arrivent à la conclusion que ce professionnel joue un rôle 
important dans le développement de relations d'amitié entre pairs ayant ou non des besoins particuliers. Ainsi, l'ergothérapeute peut contribuer à l'organisation d'un environnement scolaire favorisant les interactions sociales en suggérant des modifications à apporter aux espaces de récréation et aux règles de jeux les plus populaires. Il peut également aider les enseignants en offrant des suggestions facilitant la participation des élèves ayant des besoins particuliers aux activités de la classe. Toujours selon ces auteurs, les connaissances de l'ergothérapeute sur la nature du handicap, l'analyse de tâches et l'interaction entre la personne, son environnement et sa tâche contribuent à l'aide qu'il peut apporter à l'enseignant dans la planification de l'inclusion. Ainsi, une attention particulière peut être portée à la co-planification mettant à profit l'intérêt et les forces de ces élèves, la participation aux activités de la classe, l'expérience de réussite de même que l'interaction avec les pairs. Idol (2006) met en évidence que la collaboration entre l'enseignant et l'aide-enseignant, ainsi qu'entre l'enseignant spécialiste de l'éducation spécialisée et l'orthophoniste contribue à la mise en place de pratiques plus inclusives. Quant à Noël (2009), il rend également compte de l'apport substantiel de la collaboration entre collègues dans la mise en place de pratiques plus inclusives, notamment le co-enseignement réunissant l'enseignant de la classe ordinaire et l'enseignant spécialisé.

Avec les parents: La métasynthèse traite peu de la collaboration avec les parents. Néanmoins, Logan (2006) fait explicitement mention de la collaboration qui subsiste entre l'aide-enseignant et les parents d'élèves à risque ou ayant des besoins particuliers. Quant à Beauregard (2006), elle reconnaît l'appréciation qu'ont les enseignants de l'aide qu'apportent les parents d'enfants dysphasiques. Groom et Rose (2005) de même que Sokal (2012) soulignent l'importance d'une bonne collaboration entre l'enseignant et les parents. Cette collaboration respectueuse est aussi nécessaire entre les parents et l'aide-enseignant (Groom \& Rose, 2005).

\section{Conditions favorables en lien à la culture de collaboration}

La culture de collaboration renvoie ici à une communauté stimulante qui se démarque par ses habitudes de collaboration et d'acceptation où tous ses membres sont valorisés (Booth \& Ainscow, 2002). Deux dimensions de la culture de collaboration retiennent l'attention à l'issue de la métasynthèse, soit la collaboration au sein de la classe et la collaboration à l'extérieur de la classe. Il apparaît important de préciser que plusieurs professionnels de l'éducation sont explicitement identifiés, par les enseignants, comme vecteur de soutien aux pratiques plus inclusives. À ce titre, Idol (2006) précise qu'une équipe de travail élargie, composée d'une variété de professionnels, contribue à la qualité des enseignements et à l'adaptation ou la modification du curriculum. Paterson (2007) estime également que le soutien des spécialistes de l'éducation spécialisée contribue au développement d'une meilleure connaissance et d'une meilleure 
compréhension des élèves chez les enseignants, favorisant ainsi la mise en place de pratiques enseignantes plus adaptées aux élèves qui composent la classe. De façon similaire, Ouellet, Caya et Tremblay (2011) concluent que le travail en collaboration permet non seulement de dresser un portrait plus juste des élèves, mais aussi d'assurer une plus grande cohérence en matière d'intervention. Les professionnels mis à profit dans une perspective collaborative comprennent les enseignants ressources ${ }^{7}$, les aides-enseignants (p. ex. Idol, 2006), les experts de l'éducation spécialisée (p. ex. Paterson, 2007), les coordonnateurs responsables des services aux élèves ayant des besoins particuliers (p. ex. Burton \& Goodman, 2011), les psychologues scolaires (p. ex. Boyle et al., 2012) et l'orthopédagogue (logopédiste) (p. ex. Rousseau, 2009). Les enseignants constituent également une source importante d'expertise au cœur des pratiques collaboratives (p. ex. Sokal, 2012).

\section{Au sein de la classe}

La métasynthèse révèle que les pratiques collaboratives au sein de la classe sont associées à des pratiques inclusives plus efficaces, notamment en raison de la progression des apprentissages et d'une participation plus grande aux activités d'apprentissage chez les élèves à risque ou ceux ayant des besoins particuliers. Le co-enseignement (team teaching) constitue une forme de pratique collaborative associée aux pratiques plus inclusives mettant à profit une plus grande participation des élèves. Selon O'Rourke et Houghton (2009), il permet non seulement la collaboration entre deux enseignants, mais également le soutien mutuel. L'apport du co-enseignement est fortement associé à l'équipe pédagogique qui vise un même objectif, mais déploie différentes approches pour l'atteindre. Toujours selon O'Rourke et Houghton (2009), les élèves apprécient la présence simultanée de deux enseignants puisque cela leur permet de poser davantage de questions, tout en prenant le temps de bien comprendre les réponses. Ils se disent moins réticents à poser des questions dans ce contexte. Avec un seul enseignant dans la classe, ils avouent être embarrassés lorsqu'ils sollicitent trop d'attention, étant donné leur difficulté de compréhension - les activités d'écriture étant les plus anxiogènes à cet égard. Plus spécifiquement, pour les élèves ayant des besoins particuliers (déficience intellectuelle modérée), le co-enseignement contribuerait à diminuer le sentiment de solitude qu'ils peuvent éprouver au sein de la classe (O’Rourke \& Houghton, 2009).

Le travail en collaboration de l'enseignant et de l'orthopédagogue se révèle également une stratégie gagnante qui place l'enseignant en confiance devant la classe hétérogène, bien qu'une période d'adaptation soit nécessaire (Granger, Debeurme \& Kalubi, 2013; Rousseau \& Thibodeau, 2011). Le co-enseignement de stratégies de lecture, d'écriture, d'organisation de l'information et la co-planification de l'enseignement sont des stratégies appréciées. Cela dit, Rousseau (2009) précise qu' «une planification hebdomadaire conjointe entre l'orthopédagogue et l'enseignant disciplinaire [doit être] prévue à l'horaire» (p. 107) afin 
d'assurer la réussite de cette collaboration. Granger, Debeurme et Kalubi (2013) soutiennent que les enseignants qui œuvrent auprès d'une diversité d'élèves se sentent davantage en confiance lorsqu'ils peuvent compter sur l'aide des enseignants ressources ou des orthopédagogues, notamment pour leur apport dans la compréhension de certaines problématiques telles que les troubles du langage. Idol (2006) encourage également les pratiques collaboratives en classe, notamment entre l'enseignant et l'enseignant ressource, ce dernier possédant un bagage important en matière d'intervention spécialisée. Selon l'auteure, l'enseignant ressource devrait pouvoir soutenir au besoin l'enseignant dans sa classe ordinaire, et ce, sans obligation d'assignation formelle. Enfin, le soutien de l'aide-enseignant, lorsqu'utilisé judicieusement à l'intérieur de la classe, se révèle une autre source de collaboration importante (Idol, 2006; O'Rourke \& Houghton, 2009).

Le modelage par les collègues enseignants est une autre technique soulevée par Boyle et al. (2012) pour mieux comprendre comment enseigner à une classe hétérogène, bien qu'il oblige l'accueil d'un autre enseignant au sein de sa classe. D'ailleurs, O'Rourke et Houghton (2009) associent non seulement les pratiques collaboratives entre collègues au renouvellement des pratiques enseignantes, mais également au développement de liens d'amitié. Étant donné toute la richesse qui réside dans l'aide apportée par les différents spécialistes, il faut reconnaitre qu'il peut se révéler inconfortable, voire confrontant pour les enseignants d'accueillir quelqu'un d'autre dans leur propre classe (Granger, Debeurme \& Kalubi, 2013; Rousseau \& Thibodeau, 2011). Le défi entourant l'atteinte de cet objectif relève de la crainte du jugement de l'autre, de se faire surprendre en train de commettre une erreur et de perdre les liens de confiance avec ses élèves.

Il va sans dire que les pratiques collaboratives au sein de la classe ne sont pas sans lien aux attentes des enseignants envers les élèves. Ainsi, Mowat (2009) met en évidence la nécessité, chez les enseignants en contexte inclusif, d'avoir des attentes élevées et explicites envers tous leurs élèves. Pour ce faire, les enseignants gagnent à faire preuve de flexibilité en ayant recours aux approches différenciées qui prennent en considération les apprentissages antérieurs des élèves, tout en offrant le soutien nécessaire à la réalisation des apprentissages attendus.

\section{À l'extérieur de la classe}

Les écrits analysés font ressortir qu'une culture de collaboration au sein de l'établissement scolaire renvoie à la nécessité de briser l'isolement et de réfléchir sur ses gestes pédagogiques en lien à la diversité d'élèves qui composent la classe, et ce, tant avec ses collègues enseignants qu'avec d'autres professionnels de l'éducation.

Les coordonnateurs responsables des services aux élèves ayant des besoins particuliers (Burton \& Goodman, 2011) sont des professionnels dont le rôle vise à soutenir le processus d'inclusion des élèves. Ils travaillent de concert avec le personnel non enseignant (aide-enseignant et enseignant ressource). Les coordon- 
nateurs ont la responsabilité de coordonner les actions du personnel non enseignant en soutien aux enseignants certes, mais surtout en soutien aux élèves. Les liens que les coordonnateurs et le personnel non enseignant établissent avec les élèves ayant des besoins particuliers et leur famille contribuent au développement d'une connaissance approfondie du jeune et de son contexte. Ces connaissances gagnent à être prises en compte par les enseignants, pour ensuite être réinvesties en classe ordinaire afin que l'école puisse être un endroit sécurisant pour eux, où les adultes représentent non seulement des figures d'autorité, mais également des figures de bienveillance. L'accessibilité, la confidentialité et l'empathie des coordonnateurs et des enseignants sont des caractéristiques fondamentales dans l'aide apportée auprès de ces jeunes.

À l'intérieur des murs de l'école, l'entraide au sein du personnel enseignant est importante pour travailler efficacement auprès des élèves ayant des besoins particuliers en classe ordinaire, réitèrent Boyle et al. (2012). À ce titre, l'école secondaire gagne à décloisonner ses départements afin de promouvoir la création de liens de collaboration entre tout son personnel. Ce décloisonnement contribue à l'émergence de pratiques plus inclusives de même qu'au développement d'un climat d'école plus positif en permettant de réfléchir ensemble, selon plusieurs perspectives, sur l'enseignement et l'intervention en contexte de diversité. Les auteurs mettent en évidence que de telles pratiques collaboratives au sein de l'école secondaire permettent aux enseignants de découvrir et de mettre à l'essai une variété de stratégies d'enseignement, et ainsi de découvrir celles qui s'avèrent les plus efficaces pour répondre aux particularités de leurs classes. La culture de collaboration permet également de partager les stratégies efficaces avec ses pairs et ainsi contribuer au développement d'un plus grand sentiment de compétence. Cette capacité à collaborer et communiquer efficacement sur ses pratiques professionnelles est décrite comme étant une compétence attendue des enseignants en contexte inclusif où chaque membre doit se percevoir comme faisant partie d'une équipe multidisciplinaire où tous apportent une contribution qui traduit leurs compétences distinctes (Sokal, 2012).

Un autre aspect positif associé au travail collaboratif entre différents professionnels et les enseignants est la confiance en soi que gagnent les enseignants (Labonté, 2011). En effet, les encouragements prodigués entre eux ainsi que le travail d'équipe réalisé en juxtaposant les expertises de chacun contribuent à générer un plus grand sentiment de confiance en ses capacités d'enseigner à une diversité d'élèves. Labonté (2011) met également en évidence l'appréciation des enseignants à l'égard de ses échanges d'expertises et l'aide concrète qui en découle. De plus, Boyle et al. (2012) soulignent l'importance de mobiliser l'ensemble du personnel scolaire dans le processus d'inclusion afin de créer un environnement scolaire accueillant et efficace. Dans cette perspective, tous les personnels qui œuvrent au sein de l'école sont visés, incluant le personnel du secrétariat et la conciergerie - qui sont aussi des acteurs clés de l'inclusion scolaire. L'accueil chaleureux des nouveaux enseignants, et donc leur propre inclusion sociale au 
sein de l'école, constitue également une condition favorable au développement d'écoles plus inclusives (Sokal, 2012). Pour cet auteur, ce type d'accueil contribue à briser le sentiment d'isolement de la relève en plus de favoriser le sentiment d'aise à solliciter de l'aide afin d'optimiser ses pratiques. Comme le réitèrent Boyle et al. (2012), les enseignants ont tout à gagner de s'unir et travailler en équipe afin de trouver des solutions face aux défis rencontrés. L'énergie nouvelle qu'amènent les nouveaux enseignants ne devrait pas être sous-estimée puisqu'elle contribue à stimuler les enseignants d'expérience et les expose à de nouvelles pratiques.

Enfin, le travail de concert avec le psychologue scolaire s'avère une autre forme de collaboration qui contribue au développement de pratiques plus inclusives. L'étude de Boyle et al. (2012) révèle que son travail est essentiel afin d'assurer une expérience scolaire de qualité. La relation établie avec les enseignants se révèle aussi essentielle à son travail. À ce titre, l'animation d'un groupe de soutien réservé aux enseignants en contexte inclusif peut s'avérer une stratégie efficace, notamment lorsque ces enseignants accueillent dans leur classe des élèves ayant des besoins particuliers. Toujours selon les auteurs, de savoir qu'aucun jugement n'est associé à la demande d'aide auprès d'un collègue constitue un mécanisme puissant favorisant le travail des enseignants auprès d'une diversité d'élèves, en plus de contribuer au développement d'une meilleure compréhension de certaines problématiques vécues par leurs élèves.

\section{Discussion}

Cette métasynthèse aura permis de mettre en exergue les apports et les limites des pratiques qui tendent vers l'inclusion scolaire en enseignement primaire et secondaire. En juxtaposant ces résultats à ceux d'études adoptant une approche purement quantitative, on constate les apports des pratiques plus inclusives sur l'apprentissage (voir Dessemontet, Bless \& Morin, 2012; Tremblay, 2012) et sur la socialisation (voir De Boer, Pijl, Post \& Minneart, 2013; Koutrouba, Vamvakari \& Steliou, 2014; Litvack, 2004).

Les résultats ont également permis d'identifier une évolution dans les préoccupations des chercheurs intéressés à la thématique de l'inclusion. En effet, de moins en moins de recherches portent sur les effets de l'inclusion, alors que celles portant sur les moyens de «faire de l'inclusion» occupent une place considérable. $\mathrm{Ne}$ s'agit-il pas là d'une marque de sensibilité par les chercheurs aux réalités des praticiens qui trouvent difficile l'enseignement à une diversité d'élèves, et ce, malgré les connaissances associées à ses bienfaits chez tous les élèves qui composent la classe (voir, p. ex., Vienneau, 2004)? Outre les apports et les limites associés aux pratiques qui tendent vers l'inclusion scolaire, les résultats mettent clairement en évidence que les pratiques collaboratives sont centrales dans le développement d'écoles plus inclusives. Cette notion essentielle qu'est la 
collaboration ressortait déjà il y a plus de vingt ans comme étant un des facteurs favorisant les pratiques inclusives (Beninghof \& Singer, 1995-1996). Ainsi, la recherche récente sur l'inclusion réitère la fonction essentielle de la collaboration et précise les conditions de celle-ci. À ce titre, le travail collaboratif avec l'aide-enseignant semble constituer une piste importante à considérer dans la mise en ouvre de l'inclusion. En effet, les différents travaux recensés stipulent que l'aide-enseignant, par les différents rôles assumés auprès de l'élève et des autres acteurs de l'éducation et de la communauté, contribue à la qualité du soutien envers les élèves et permet une meilleure concertation entre acteurs scolaires (Groom \& Roise, 2005; Logan, 2006). Comme l'aide-enseignant est tantôt présent ou absent des systèmes scolaires (particularité nationale), il devient nécessaire de réfléchir non seulement sur "comment" faire l'inclusion, mais également sur les ressources humaines à mobiliser pour y arriver. Bien que la présente métasynthèse fasse peu état de la complexité associée à la collaboration, voire du peu de place qu'occupent les parents au sein de cette collaboration, cet aspect pourrait faire en soi l'objet d'une autre métasynthèse. Il pourrait aussi être intéressant de mieux documenter et mettre en évidence la complémentarité des actions posées par les différents professionnels de l'éducation en contexte d'inclusion scolaire.

\section{Conclusion}

Les résultats de l'étude présentée ici renvoient à l'importance du travail collaboratif au sein des écoles désireuses d'adopter des pratiques plus inclusives. La métasynthèse révèle que la collaboration entre tous les acteurs scolaires semble associée à des retombées positives tant pour les enseignants et les autres professionnels de l'éducation que pour les élèves. Cela dit, pour transformer l'école en un réel lieu de collaboration, soit un lieu d'apprentissage plutôt qu'un lieu de rassemblement d'acteurs sous un même toit (pour reprendre l'idée de Rogers, 2007), un solide leadership de la direction d'école est nécessaire, notamment en favorisant un contexte favorable aux échanges pédagogiques et en valorisant l'ouverture au changement et l'adaptation ou l'exploration de nouvelles façons de faire (Rousseau \& Point, 2015; Thibodeau et al., 2016). Dès lors, il convient d'admettre que l'enseignement à une diversité d'élèves ne peut pas reposer que sur l'expertise des spécialistes de l'adaptation scolaire, mais doit plutôt mettre à profit les savoirs professionnels des différents acteurs qui composent l'école (savoirs spécialisés, savoirs disciplinaires, savoirs didactiques, savoirs psychologiques, etc.). Les apports associés aux pratiques plus inclusives sur l'apprentissage, la socialisation, l'engagement et le sentiment d'appartenance des jeunes envers l'école méritent qu'une culture de collaboration se déploie, et ce, malgré les défis que peuvent engendrer le travail en collaboration de même que les changements qu'une telle perspective de travail est susceptible d'occasionner. 
En terminant, il apparait judicieux d'ouvrir sur quelques pistes de recherche susceptibles de pousser encore plus loin les résultats présentés ici. Considérant la place indéniable qu'occupe la collaboration de l'enseignant avec les autres professionnels de l'éducation au sein même de la classe, quelles sont les conditions favorables ou défavorables à la mise en œuvre d'une telle collaboration? Comment se décline la collaboration entre les parents et les professionnels de l'éducation en contexte inclusif? Cette collaboration se manifeste-t-elle de la même façon entre les parents et les enseignants? La formation initiale et continue peut-elle jouer un rôle tangible dans le développement de compétences collaboratives? Enfin, quels changements organisationnels peuvent faciliter le développement d'habitudes de co-planification et de co-enseignement en contexte inclusif?

\section{Notes}

1 Projet de recherche subventionné par le Fonds québécois de recherche - Société et culture (FRQSC) et le Ministère de l'Éducation, du Loisir et du Sport [MELS].

2 L'appellation "élèves à risque» renvoie à la définition du Ministère de l'Éducation, du Loisir et du Sport (MELS, 2007, p. 24), qui précise qu'il s'agit d'élèves «du préscolaire, du primaire et du secondaire qui présentent des facteurs de vulnérabilité susceptibles d'influer sur leur apprentissage ou leur comportement». Les élèves à risque comprennent ceux ayant des difficultés d'apprentissage ou présentant des troubles du comportement.

3 L'appellation «élèves ayant des besoins particuliers» renvoie plutôt aux élèves ayant des troubles graves de comportement, une déficience intellectuelle profonde, moyenne ou sévère, une déficience motrice légère ou grave, une déficience organique, une déficience langagière, une déficience visuelle, une déficience auditive, des troubles envahissants du développement et des troubles relevant de la psychopathologie (MELS, 2007).

4 Comme ce projet de recherche est subventionné par le FRQSC et le MELS, c'est la définition du Ministère qui a été retenue pour désigner les élèves à risque et les élèves ayant des besoins particuliers (voir notes de fin).

5 L'utilisation de la parenthèse désigne les résultats en lien aux élèves ayant des besoins particuliers.

6 Plusieurs appellations sont utilisées pour désigner cette ressource qui œuvre directement auprès des élèves mais en soutien aux enseignants (p. ex. auxiliaire de vie scolaire en France - Toullec-Théry \& Nédélec-Trohel, 2010 -, teacher aid dans certaines provinces canadiennes et états américains).

7 Dans le texte d'Idol (2006) et dans d'autres textes consultés, l'enseignant ressource peut désigner un enseignant d'expérience ayant des connaissances en éducation spécialisée, voire des spécialistes œuvrant à titre d'enseignants ressources (par exemple, l'orthophoniste).

\section{Références}

AuCoin, A. (2010). Pas plus spécial que nécessaire: étude de l'historique, du cadre légal et du vécu de l'inclusion scolaire chez les Acadiens et les Acadiennes de la Nouvelle-Écosse (Thèse de doctorat inédite). Université de Moncton.

AuCoin, A. \& Vienneau, R. (2014). L'inclusion scolaire et la dénormalisation. Proposition d'un nouveau paradigme. Dans N. Rousseau (Éd.), La pédagogie de l'inclusion scolaire (3e éd. revue et augmentée, pp. 65-88). Québec, Québec: Presses de l'Université du Québec.

Beaucher, V. \& Jutras, F. (2007). Étude comparative de la métasynthèse et de la méta-analyse qualitative. Recherches qualitatives, 27, (2), 58-77. 
Beauregard, F. (2006). Représentations sociales des parents et des enseignants de leurs rôles dans l'intégration scolaire d'un élève dysphasique en classe ordinaire au primaire. Revue des sciences de l'éducation, 32, (3), 545-565.

Bélanger, N. \& Duchesne, H. (Éd.) (2010). Des écoles en mouvement. Inclusion d'élèves en situation de handicap ou éprouvant des difficultés à l'école. Québec: Presses de l'Université du Québec.

Beninghof, A.M. \& Singer, A.-L.F. (1995-1996). Ideas for inclusion. The School Administrator's Guide. Longmont, CO: Sopris West.

Bergeron, G. \& St-Vincent, L.-A. (2011). L'intégration scolaire au Québec: regard exploratoire sur les défis de la formation à l'enseignement au primaire et préscolaire. Éducation et francophonie, 39, (2), 272-295.

Boer, A. de, Pijl, SJ., Post, W. \& Minnart, A. (2012). Which Variables Relate to the Attitudes of Teachers, Parents and Peers towards Students with Special Educational Needs in Regular Education? Educational Studies, 38, (4), 433-448.

Booth, T. \& Ainscow, M. (Éd.) (2002). Index for inclusion: Developing learning and participation in schools. Bristol, Royaume-Uni: Centre for Studies on Inclusive Education.

Bourdieu, P. (Éd.). (1993). La misère du monde. Paris, France: Édition de Minuit.

Boyle, C., Topping, K., Jundal-Snape, D. \& Norwich, B. (2012). The Importance of Peer-Support for Teaching Staff when Including Children with Special Educational Needs. School Psychology International, 33, (2), 167-184.

Burton, D. \& Goodman, R. (2011). Perspectives of SENCos and support staff in England on their roles, relationships and capacity to support inclusive practice for students with behavioural emotional and social difficulties. Pastoral Care in Education, 29, (2), 133-149.

Campbell, R., Pound, P., Pope, C., Britten, N., Pill, R., Morgan, M. \& Donovan, J. (2003). Evaluating meta-ethnography: a synthesis of qualitative research on lay experiences of diabetes and diabetes care. Social Science \& Mecidine, 56, 671-684.

Combs, S., Elliott, S. \& Whipple, K. (2010). Elementary Physical Education Teachers' Attitudes towards the Inclusion of Children with Special Needs: A Qualitative Investi-gation. International Journal of Special Education, 25, (1), 114-125.

Crahay, M. (2000). L'école peut-elle être juste et efficace? De l'égalité des chances à l'égalité des acquis. Bruxelles, Belgique: De Boeck Université.

Dessemontet, R.S., Bless, G. \& Morin, D. (2012). Effects of inclusion on the academic achievement and adaptive behaviour of children with intellectual disabilities. Journal of Intellectual Disability Research, 56, (6), 579-587.

Dubé, F. (2008). Élèves en difficulté diapprentissage en classe ordinaire: analyse de projets de services innovateurs au primaire (Thèse de doctorat inédite). Université de Montréal.

Erwin, E.J., Brotherson, J. \& Summers, J.A. (2011). Understanding Qualitative Metasynthesis: Issues and Opportunities in Early Childhood Intervention Research. Journal of Early Intervention, 33, (3), 186-200.

Fougeyrollas, P. (2010). La funambule, le fil et la toile. Transformations réciproques $d u$ sens $d u$ handicap. Québec: Presses de l'Université Laval.

Gallucci, C. (1997). The MESH Manual for inclusive schools. Projet MESH: Making effective schools happen for all students. Washington Office of the State Superintendent of Public Instruction. Olympia (ERIC Document Reproduction Service no ED450 535).

Gardou, C., Poizat, D. \& Audureau, J.-P. (2004). L'école entre catégorisation et inclusion. Aux marges du palais, les enfants en situation de handicap. Revue des sciences humaines et sociales, 103, 25-40.

Granger, N., Debeurme, G. \& Kalubi, J.-C. (2013). Les cercles d'apprentissage et d'inclusion: regard sur la transformation des pratiques enseignantes au secondaire. Éducation et francophonie, 41, (2), 232-248.

Groom, B. \& Rose, R. (2005). Supporting the inclusion of pupils with social, emotional and 
behavioural difficulties in the primary school: the role of teaching assistants. Journal of Research in Special Educational Needs, 5, (1), 20-30.

Idol, L. (2006). Toward inclusion of special education students in general education: a program evaluation of eight schools. Remedial and Special education, 27, (2), 77-94.

Kartsen, S., Peetsma, T., Roeleveld, J. \& Verger, M. (2001). The Dutch Policy of Integration put to the Test: Differences in Academic and Psychosocial Development of Pupils in Special and Mainstream Education. European Journal of Special Needs Education, 16, (3), 193-205.

Kelly, N. \& Norwich, B. (2004). Pupils' perceptions of self and of labels: Moderate learning difficulties in mainstream and special schools. British Journal of Educational Psychology, 74, (3), 411-435.

Koutrouba, K., Vamvakari, M. \& Steliou, M. (2006). Actors Correlated with Teachers' Attitudes towards the Inclusion of Students with Special Educational Needs in Cyprus. European Journal of Special Needs Education, 24, (4), 381-394.

Labonté, M. (2011). Évaluation de la perception des intervenants de la mise en auvre d'un modèle d'équipe de soutien à l'enseignant pour intégrer les services à l'école en contexte inclusif (Thèse de doctorat inédite). Université de Montréal.

Lebeaume, J. \& Coquidé, M. (2002). Hétérogénéité et différenciation. Aster, 35, 3-15.

Lee, L. \& Low, H. (2013). «Unconscious» inclusion of students with learning disabilities in a Malaysian mainstream primary school: Teachers' perspectives. Journal of Research in Special Educational Needs, 13, (3), 218-228.

Littell, J.H., Campbell, M., Green, S. \& Toews, B. (2008). Screening and data extraction forms. Dans J.H. Littell, J. Corcoran \& V. Pillai (Éd.), Systematic reviews and meta-analysis (pp. 160-171). New York: Oxford University Press.

Litvack. M.S. (2004). High-and Average-Achieving Children's Attitudes Toward Classmates with Disabilities (Thèse de doctorat inédite). Université McGill.

Logan, A. (2006). The Role of the Special Needs Assistant Supporting Pupils with Special Educational Needs in Irish Mainstream Primary Schools. Support for Learning, 21, (2), 92-99.

Ministère de l'Éducation, du Loisir et du Sport (2007). L'organisation des services éducatifs aux étudiants à risque et aux étudiants handicapés ou en difficulté d'adaptation ou d'apprentissage (EHDAA). Québec: Gouvernement du Québec.

Morefield, J. (2002). Transforming education: recreating schools for all children. [En ligne, consulté le 3 octobre 2009]. www.newhorizons.org/article.morefield.html

Morrison, R. \& Burgman, I. (2009). Friendship experiences among children with disabilities who attend mainstream Australian schools. Canadian Journal of Occupational Therapy, 76, (3), 145-152.

Morvan, J.-S. (2015). Repenser l'échec et la réussite scolaire. Issy-les-Moulineaux, France: ESF éditeur.

Mowat, J. (2009). The Inclusion of Pupils Perceived as Having Social and Emotional Behavioural Difficulties in Mainstream Schools: A Focus upon Learning. Support for Learning, 24, (4), 159-169.

Noël, I. (2009). À qui profite l'intégration? Intégration scolaire d'enfants en situation de handicap: perception par les enseignantes et les enseignants titulaires des apports pour les autres enfants de la classe. Formations et pratiques d'enseignements en question, (9), 177-197.

Nootens, P. (2010). Étude descriptive de pratiques exemplaires diadaptation de lienseignement en contexte diinclusion d'élèves en difficultés langagières au primaire (Thèse de doctorat inédite). Université de Sherbrooke.

Nowicki, E.A. \& Brown, J.D. (2013). «A Kid Way»: Strategies for Including Classmates with Learning or Intellectual Disabilities. Intellectual and Developmental Disabilities, 51, (4), 253-262. 
O'Rourke, J. \& Houghton, S. (2009). The Perceptions of Secondary Teachers and Students about the Implementation of an Inclusive Classroom Model for Students with Mild Disabilities. Australian Journal of Teacher Education, 34, (1), 23-4.

Ouellet, S., Caya, I. \& Tremblay, M.-P. (2011). L'apport d'une communauté d'apprentissage pour développer des pratiques collaboratives et inclusives: une recherche-action. Éducation et francophonie, 39, (2), 207-226.

Paterson, D. (2007). Teachers' in-flight thinking in inclusive classrooms. Journal of Learning Disabilities, 40, (5), 427-435.

Petticrew, M. \& Roberts, H. (2006). Systematic Reviews in the Social Sciences: A practical guide. Oxford: Blackwell Publishing.

Picon, I. (2010). Milieux spécialisé ou ordinaire: conséquences sur le processus d'adolescence des jeunes ayant une déficience intellectuelle. Revue francophone de la déficience intellectuelle, 21, 66-78.

Pilay, J., Dunbar-Krige, H. \& Mostert, J. (2013). Learners with behavioural, emotional and social difficulties' experiences of reintegration into mainstream education. Emotional \& Behavioural Difficulties, 28, (3), 310-326.

Rogers, C. (2007). Experiencing an «inclusive» education: Parents and their children with special educational needs. British Journal of Sociology of Education, 28, (1), 55-68.

Rousseau, N. (2009). Conditions de mise en œuvre d'une pédagogie inclusive. Le cas de la Gaspésie. Formation et pratiques d'enseignement en questions. Formations et pratiques d'enseignements en question, (9), 97-115.

Rousseau, N., Bergeron, G. \& Vienneau, R. (2013). L'inclusion scolaire pour gérer la diversité: des aspects théoriques aux pratiques dites efficaces. Revue suisse des sciences de l'éducation, 35, (1), 71-88.

Rousseau, N. \& Point, M. (2015). Les enjeux de l'intégration et de l'inclusion scolaires des élèves à risque du primaire et du secondaire: méta-analyse et méta-synthèse [Rapport de recherche déposé au FRQSC]. Trois-Rivières, Québec: Chaire de recherche Normand-Maurice, Université du Québec à Trois-Rivières.

Rousseau, N. \& Thibodeau, S. (2011). S'approprier une pratique inclusive: regard sur le sentiment de compétence de trois équipes écoles au cœur d'un processus de changement. Éducation et francophonie, 39, (2), 145-164.

Slee, R. \& Weiner, G. (2001). Education reform and reconstruction as a challenge to research genres: Reconsidering school effectiveness research and inclusive schooling. School Effectiveness and School Improvement, 12, (1), 83-98.

Sokal, L. (2012). What are schools looking for in new, inclusive teachers? McGill Journal of Education, 47, (3), 403-420.

Talbot, L. (2011). Prendre en compte la diversité des élèves. Ressources ou défis pour les enseignants? Dans L. Talbot (Éd.), L'hétérogénéité: maîtres et élèves (pp. 7-12). Toulouse, France: Presses universitaires du Mirail.

Thibodeau, S., Gélinas-Proulx, A., St-Vincent, L.-A., Leclerc, M., Labelle, J. \& Ramel, S. (2016). La direction d'école: un acteur crucial pour l'inclusion scolaire. Dans L. Prud'homme, H. Duchesne, P. Bonvin et R. Vienneau (Éd.), L'inclusion scolaire: ses fondements, ses acteurs et ses pratiques (pp. 57-70). Bruxelles: De Boeck Supérieur.

Toullec-Théry, M. \& Nédélec-Trohel, I. (2010). Interactions entre un professeur, un AVS et un élève handicapé en classe pour l'inclusion scolaire (CLIS). Carrefours de l'éducation, 1, (29), 161-180.

Tremblay, P. (2012). Évaluation comparée de deux dispositifs scolaires destinés à des élèves ayant des troubles d'apprentissage, Revue française de pédagogie, 2, (179), 63-72.

Vienneau, R. (2004). Impacts de l'inclusion scolaire sur l'apprentissage et le développement social. Dans N. Rousseau et S. Bélanger (Éd.), La pédagogie de l'inclusion scolaire (pp. 125-152). Québec: Presses de l’Université du Québec. 
Vienneau, R. (2010). Les effets de l'inclusion scolaire: une recension des écrits (2000 à 2009). Dans N. Rousseau (Éd.), La pédagogie de l'inclusion scolaire. Pistes d'action pour apprendre tous ensemble (2e éd.) (pp. 237-263). Québec: Presses de l'Université du Québec.

Walsh D. \& Downe, S. (2005). Meta-synthesis method for qualitative research: a literature review. Journal of Advanced Nursing, 50, (2), 204-211.

Mots-clés: Enseignement, inclusion scolaire, pratiques collaboratives, élèves ayant des besoins particuliers, conditions favorables

\section{Die Vorteile und Beschränkungen der inklusiven Praxis, sowie die Rolle der Zusammenarbeit im praktischen Bereich der Inklusion: eine Meta-Synthese}

\section{Zusammenfassung}

Dieser Artikel stammt aus den Ergebnissen einer Meta-Synthese, deren Hauptziel es war, ein neues Verständnis für die Problematik der Integration und schulischen Inklusion von Schülerinnen und Schülern mit besonderen Bedürfnissen in regulären Klassen der Grundschule und des Gymnasiums zu schaffen. In diesem Artikel werden zuerst die Ergebnisse der Meta-Synthese in Bezug auf Beiträge und Einschränkungen schulischer Inklusion betrachtet. Anschliessend geht es um die kollaborative Praxis, die eine wesentliche Voraussetzung für die Entwicklung der inklusiven Praxis ist. Die drei Dimensionen der Zusammenarbeit werden näher erläutert - es sind dies die Zusammenarbeit mit und zwischen Arbeitspartnern, die Zusammenarbeit mit und zwischen Expertinnen und Experten, sowie die Zusammenarbeit mit Eltern.

Schlagworte: Lehre, Schulische Integration, Kollaborative Praxis, Schüler mit besonderen Bedürfnissen, Güngstige Umstände 


\section{Vantaggi e limiti relativi alle pratiche inclusive e ruolo della collaborazione in queste pratiche : una meta-sintesi}

\section{Riassunto}

Questo articolo scaturisce dai risultati di una meta-sintesi che aveva come principale obiettivo quello di produrre una nuova comprensione dei problemi inerenti l'integrazione e l'inclusione scolastica degli alunni con bisogni speciali nelle classi regolari di primaria e secondaria. L'articolo presenta, in primo luogo, i risultati della meta-sintesi tenendo conto dei vantaggi e dei limiti associati alle pratiche che favoriscono l'inclusione scolastica. In secondo luogo, l'articolo si interessa alle pratiche collaborative, essenziali per lo sviluppo di pratiche piu' inclusive. Tre dimensioni della collaborazione sono esplorate, la collaborazione con e tra i pari, la collaborazione con e tra i professionisti, e la collaborazione con i genitori.

Parole chiave: Insegnamento, inclusione scolastica, pratiche collaborative, alunni con bisogni particolari, condizioni favorevoli

\section{Contributions and Limitations of Inclusive Practices and Role of Collaboration in these Practices: a Metasynthesis}

\section{Summary}

This article is based on the results of a metasynthesis whose main objective was to provide a new understanding of issues related to the integration and inclusion of students with special needs in a regular classroom at both elementary and secondary levels. Firstly, the article presents results of the metasynthesis that focus on the contributions and limitations associated with practices that tend towards inclusive education. Secondly, it focuses on collaborative practices, an essential component in the development of more inclusive practices. Three dimensions of collaboration are explored: collaboration with and by peers, collaboration with and among professionals, and collaboration with parents.

Keywords: Teaching, inclusion, collaborative practices, students with special needs, favorable conditions 